\title{
Optimized low computational algorithm for elderly fall detection based on machine learning techniques.
}

\author{
Sheryl Oliver $\mathrm{A}^{\mathbf{1}^{*}}$, Anuradha $\mathbf{M}^{\mathbf{1}}$, Jean Justus $\mathrm{J}^{\mathbf{1}}$, Maheshwari $\mathbf{N}^{\mathbf{2}}$ \\ ${ }^{1}$ St. Joseph's College of Engineering, Chennai, Tamil Nadu, India \\ ${ }^{2}$ SCSE, VIT University, Chennai Campus, Chennai, Tamil Nadu, India
}

\begin{abstract}
Falls are a common and often devastating problem among elder people, causing a tremendous amount of morbidity, mortality. So mechanisms to detect and avoid falls are necessary to improve common living of aged people. By developing preventing mechanisms it serves as an important factor to reduce injuries, hospitalization morbidity and mortality among older people. They are several algorithms established in fall detection (FD) and fall prevention (FP) process using feature extraction and classification algorithms. In this paper a fall detection technique is developed based on the data sets of electromyography (EMG) and electrocardiogram (ECG). The objective of this paper is to detect falls by processing with several feature extraction and classification technique for normal as well as sick people. It also focuses on the improvement of the accuracy in detecting falls. The feature extraction algorithm chosen is capable of sensing, processing and communicating the fall event under real life conditions. A hybrid classification algorithm is also proposed to achieve accuracy in detection by tracking objects and having the ability to handle the causes. The combination of numerical data is used in order to detect fall with high accuracy and reliability. The advantages of this proposed method is less computation complexity and improves efficiency of fall detection compared to existing machine learning algorithms.
\end{abstract}

Keywords: Fall, Fall prevention, Fall detection, Electromyography, Electrocardiogram, Feature extraction, Machine learning, Classification.

Accepted on November 21, 2018

\section{Introduction}

Falls are measured as a main risk in health issues among elderly people which causes increases in the mortality, morbidity rate and also it is a source of loss in autonomy now it lead to a significant impacts on national health system. According to WHO [1] $40 \%$ of people above $65 \mathrm{y}$ of age tend to fall at least once a year. This fall causes serious injuries and hospitalization rate to increases by an average of $2 \%$ until 2030 [2]. The fear of falling among elders has been increasing so the confidence level of living independent is decreasing. Records show about $3 \%$ of fallers lie without an external support and are unable to get up by themselves. Hence automatic notification to their family or caregivers after fall will be a helpful to provide an immediate medical care. After fall injuries include broken bones, abrasions to the skin and soft tissue damage $[3,4]$. The development of wireless network and sensing technologies provide the realization of context awareness in wireless body area network (WBAN). These sensors provide a central device to collect raw data from the human body. Fall detection would reduce the time between fall and arrival of medical help [5] this is realized by an automatic fall detection system through a personal emergency response system (PERS). The process of detecting and preventing fall is a challenging task. They are different fall detection approaches such as smart clothing, smart phones, smart watches and activity trackers. Recent systematic reviews highlights many proposed technology including the indication of false alarms and their cost effectiveness [6,7] these approaches uses of additive sensors such as a accelerometers, gyroscopes or ambient sensors. Previous techniques also include ADL as falling down with both legs straight or with knee flexion $[8,9]$. The popular techniques that utilizes image processing and accelerometer sensors. The basic application for detecting fall is the daily activity monitoring which is done by biological signals, body posture. The number of proposed fall detection systems developed has increased dramatically [10]. The various algorithms primarily improved the performance based on various parameters such as sensitivity, specificity and accuracy. Wearable devices based on inertial sensors have been explored, which vary in sensor type, placement, device quantity and approach. One of main challenges for wearable fall detection is the lack of research work in these areas. Sensor placement positions vary, and include waist, wrist, hip, and trunk attachment. The electrocardiogram (ECG) and electromyography (EMG) data are used. This data are largely monitored of patients over $60 \mathrm{y}$ old. ECG [11] monitoring is beneficial for cardiovascular diseases and their applications are 
for real life activities. Accordingly most of the wearable and ambient sensors include monitoring of ECG signals. EMG data can be affected by movement artifacts, physical pressure and muscle fatigue [12]. Various survey had found that EMG signals could be successful for gesture recognition $[4,13,14]$.

This EMG is used to detect the muscle activities during the daily activities of human. To achieve the daily monitoring process and the detection of fall it is quite important to distinguish falls [15]. An alarm is provided to the caregivers to give accurate help for the elder during the fall. Figure 1 shows how the machine learning techniques is classified and it describes the different algorithms used in the calculation of various process.

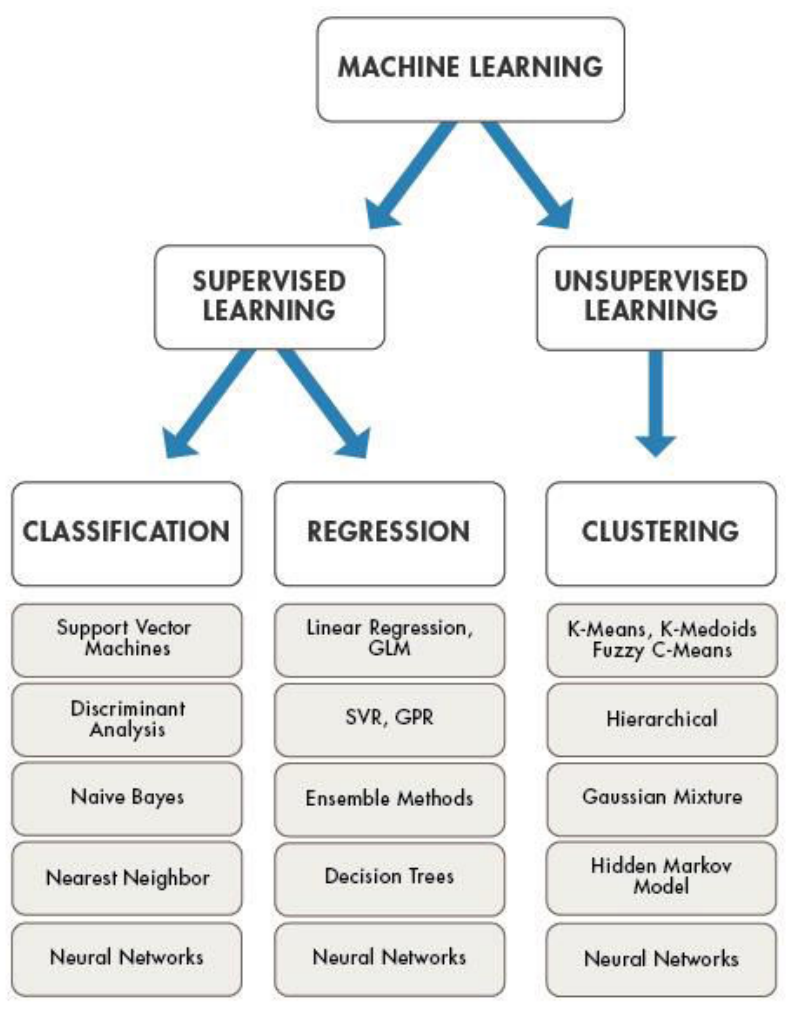

Figure 1. Machine learning.

Based on EMG and ECG a machine learning classification technique is used to identify whether the elderly can be prevented from fall. This is done by collecting dataset from the wearable electrodes and split them into training and test data set. The classification algorithm classifies the activities of the elderly people based on fall movements. By detecting those movements the elderly fall is found and the data is run through the machine learning classification algorithms to confirm fall. The various classification algorithms are support vector machine (SVM), K nearest neighbor (KNN), Navies Bayes and decision trees. In SVM the human joint measurements has been utilized that provided good performance [11]. The activities of daily living and the fall detection algorithms developed in the existing systems were not much efficient as it was implemented using the microcontroller unit, random access memory and clock speed $[16,17]$.

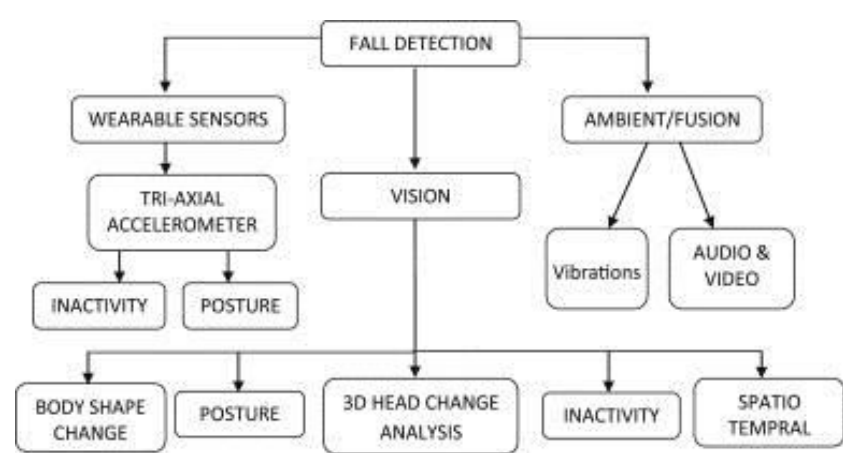

Figure 2. Fall detection approach.

The Figure 2 shows how the falls are detected using different sensors and it also depicts the classification of the fall detection mechanisms based on the human activities and posture. The machine learning process is classified into supervised and unsupervised learning techniques [18]. The support vector machine is a supervised learning model that classifies the data used based on regression analysis. The models using machine learning have been taken with six activities using motion capture [7]. Few techniques focus on the fault tolerance of new methods. In this work EMG and ECG data collected is scaled and features are extracted and then classified using few classification techniques which will be discussed later in the paper. The classification accuracy suggests which technique can be used to detect and prevent elderly fall.

\section{Related Work}

The following is the detailed descriptions of the works related to the fall detection techniques.

\section{Fall detection systems based on inertial measurement unit}

Fall detection systems are based on sensors is given by the context aware system (CAS) [7]. This system is integrated with vision based and ambient based systems that include cameras and microphones. Like the wearable FDS the CAS architecture easily provides the communication interface and the monitoring process is carried out in a low cost. Similarly the Smartphone-based architecture benefits from the commercial device that integrates with an inertial measurement unit (IMU) it supports multi interface wireless communication and the methods are specified in Table 1. They considered the mobility datasets that obtained wearable IMU that include falls from the monitored users. The collected data sets are from the experimental subjects from mobility sensors mainly accelerometers that are attached to human body. The samples were measured by wearing these devices during the daily life.

\section{Related work on wearable fall detection}

Different wearable devices that are based on inertial sensors have been used that vary in the sensor type, quantity and approach. The majority of these devices are classified into three main categories such as threshold-based, combined with 
phase and machine learning [19]. The threshold based approach uses single and multiple thresholds to extract features. Wearable fall detection also used wireless sensor network to enhance the monitoring of the elderly daily activities. The disadvantage of this system is the process uses only acceleration to detect many false positives. The researchers had combined the study on accelerometers with gyroscopes to accurately detect the fall.

Table 1. Sensors in Mobifall.

\begin{tabular}{|c|c|c|}
\hline Sensor & & Measurements recorded in "MobiFall" dataset \\
\hline Accelerometer & & Acceleration force along the $x-z$ axis $\left(\mathrm{m} / \mathrm{s}^{2}\right)$ \\
\hline Gyroscope & & Rate of rotation around the $x-z$ axis $(\mathrm{rad} / \mathrm{s})$ \\
\hline $\begin{array}{l}\text { Orientation } \\
\text { based) }\end{array}$ & (software & $\begin{array}{l}\text { Angle around the } z \text { (azimuth), } y \text { (pitch) and } x \text { (roll) } \\
\text { axis in degrees }\end{array}$ \\
\hline
\end{tabular}

\section{Ambient device based approaches}

This device based approach combines the effort of audio and visual data to detect falls through vibrational data [6]. The combination of these data produces an audiovisual signal that is taken through the environmental sensors. The process of detecting falls using this event sensing devices that collects data from the vibration is associated with the changes as well and it needs monitoring and tracking of the people. This ambient data was also useful for localization process [10].

\section{Camera based approach}

The camera based approach is based on the vision dependent recognition systems. This process as a advantage of locating people using camera, placed on overhead positions to monitor the daily activities. This approach leads to the development of various image examining process such as spatiotemporal features and 3D head position analysis.

- The spatiotemporal feature technique is used to identify different human activities from several events and provides the essential information

- 3D head position analysis is an evaluation that is dependent on head monitoring process that determines large movement inside the vision based sequence. Even different state models are used to monitor this head position using the magnitude information [20].

\section{Least square method (LSM)}

In LSM two average vectors of the classes are taken to correspond the fall and the activities of the daily living [5]. The test vector taken is compared with the reference vector, the sum of the squared difference between the vectors is calculated.

\section{Bayesian decision making (BDM)}

It is a widely used robust approach used in the statistical classification technique. The parameters are considered as mean and the training vectors [4] of each class is the covariance matrix $C$. the values are calculated based on the training records of the two classes.

\section{Fall detection flow}

The fall detection process in the existing works focuses on the indoor activities which describes the incidents of fall and different postures (lying and standing) and the events such as fall down and lying down events. This technique leads to false alarms on the incidents of fall because the postures show the similar events. The transitions time between the postures is less than the threshold value that depicts the higher probability of falling down.

\section{Methodology}

\section{Data collection}

The data collection [21] is a process of collecting different data from various subjects or source. The collected data are recorded in a systematic pattern. The formal procedure must be followed to focus on the accuracy and validity of the data. First process is the identification of necessary details regarding fall events. The collected data must determine the coordinates of fall, which electrodes give maximum difference between stable and fall. The next step is to identify whether the collected data is without outliers and hence it should be normalized. From the collected data noise is removed and only the useful values are used to derive the output.

\section{Machine learning}

Fall detection (FD) and fall prevention (FP) are a well-defined model in order to detect and prevent falls. The model is done by testing the performance of the input data. This technique depends on algorithm to calculate the results. The process begins with the classification of wearable electrodes based approach. Figure 3 describe the general model of the machine learning process of FD and FP systems.

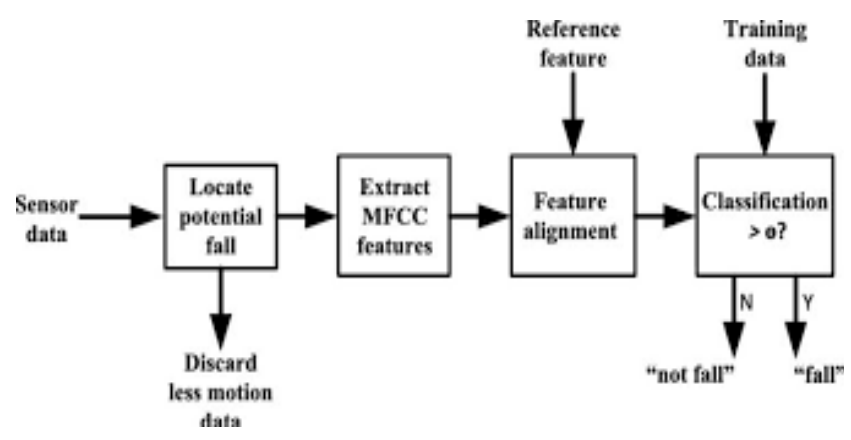

Figure 3. Model of FD and FP system.

Before describing the general model of FD and FP system the key terms is important

- Dataset

- Feature

- Instance

- Class 


\section{Feature extraction}

The feature extraction technique is also referred as dimensionality reduction (i.e.) the given input data is been reduced to an average data set by removing the redundant features. The reduction process lowers the subsequent modules [22]. Only some data needs the process of feature extraction other data sets remains unchanged. It is technique of collecting relevant attributes from the collected data. In fall system the acceleration and the angular magnitudes are extracted. In this paper two feature extraction techniques are selected and they are principle component analysis and singular value decomposition. These two techniques are selected because it provides more accuracy and efficiency compared with the existing technologies. These algorithms are commonly used for removing the redundant data and noisy data from the overall datasets. Feature extraction technique is useful in extracting a huge amount of data.

\section{Classification}

The goal of classification is to accurately predict the target class for each case. Classification is the problem of identifying to which of a set of categories. In order to evaluate any technique, we also summarize various classification algorithms such as decision trees, Naive Bayes, K-nearest neighbor and SVM. Two machine learning techniques are also discussed to understand classification algorithm easier [23].

Supervised learning: The training data are accompanied by labels indicating the class of observations. New data is classified based on the training set [24].

Unsupervised learning: The class label of the training set is unknown. Given a set of observations and measurements with the aim of establishing the existences of class. It generally takes 3 important aspects

- Ease to interpret output

- Calculation time

- Predictive power

A good classifier is required to have high scores in each of the three components, sensitivity, specificity and accuracy. The performance of the classifier in terms of accuracy, sensitivity and specificity [24].

Table 2. Performance of classifiers.

\begin{tabular}{llll}
\hline Classification techniques & Accuracy & Sensitivity & Specificity \\
\hline SVM & $80.30 \%$ & $79.40 \%$ & $79.60 \%$ \\
\hline KNN & $84 \%$ & $85 \%$ & $84.50 \%$ \\
\hline NAVIES BAYES & $84 \%$ & $83 \%$ & $86 \%$ \\
\hline
\end{tabular}

The Table 2 shows performance of classifiers with accuracy, sensitivity and specificity values of various classification techniques.

\section{Support vector machine (SVM)}

Support vector machine is a supervised learning algorithm method. To classify the dataset geometric margin is used that are separable by linear plane and found by maximizing the margin of the two classes [25]. SMO provides an efficient way for solving the derivation of support vector machine. The parameters $w$ and $b$ are solved using Lagrange multiplier method as shown in Equation 1.

$\mathrm{L}(\mathrm{w}, \mathrm{b}, \alpha)=1 / 2\|\mathrm{w}\|^{2}-\Sigma \alpha_{\mathrm{i}}\left(y_{\mathrm{i}}\left(w^{\mathrm{T}} x+b\right)-1\right) \rightarrow(1)$

SMO algorithm is used inside the WEKA tool to classify fall and the activities of daily living in the implementation of SVM.

\section{Decision tree}

A popular machine learning algorithm that consists of nodes and branches that connects nodes. The top node is defined as root node, the bottom node indicates the classes. The nodes except leaves are defined as the decision nodes. The training dataset are split into classes based on the attributes. There are several algorithm based on the decision tree among that $\mathrm{C} 4.5$ is a typical algorithm. It is an extension of ID3 algorithm that uses Shannon's entropy [26] for selecting the significant features as shown in Equation 2.

$S=\sum_{i=1}^{C}-p_{i} * \log _{2}\left(p_{i}\right)$

\section{Navies Bayes}

Navies Bayes classifier [27] is a simple probabilistic classifier based on applying Bayesian theorem. It is trained very efficiently in a supervised learning. A probabilistic framework for solving classification problems. It is easy and fast to predict class of test data. It also performs in multiclass prediction. The output of the Navies Bayes classifier shows the instance of the attributes. All this falling attributes have a probability distribution (Figure 4). Predictions are made using Bayes theorem through Equation 3.

$P(h \mid d)=(P(d \mid h) \times P(h)) / P(d) \rightarrow(3)$

Where:

- $\quad P(h \mid d)$ is the probability of $h$ given the data $d$. This is called the posterior probability.

- $\quad P(d \mid h)$ is the probability of data $d$ given that the $h$ was true.

- $P(h)$ is the probability of $h$ being true. This is called the prior probability of $h$.

- $P(d)$ is the probability of the data

\section{Proposed System}

To detect the fall on elderly person of $65 \mathrm{y}$ age and above. Initially the datasets are collected from wearable electrodes of different subjects. The pre-processing of input data set is being carried out to reduce the given data to an average data set. Fall is detected for two modules of data such as the ECG and EMG 
dataset. From the existing feature extraction techniques the data sets were applied to most of the algorithm and two extraction techniques principle component analysis (PCA) and singular value decomposition (SVD) are identified. The proposed algorithm focus on fall detection as well as prevention process.

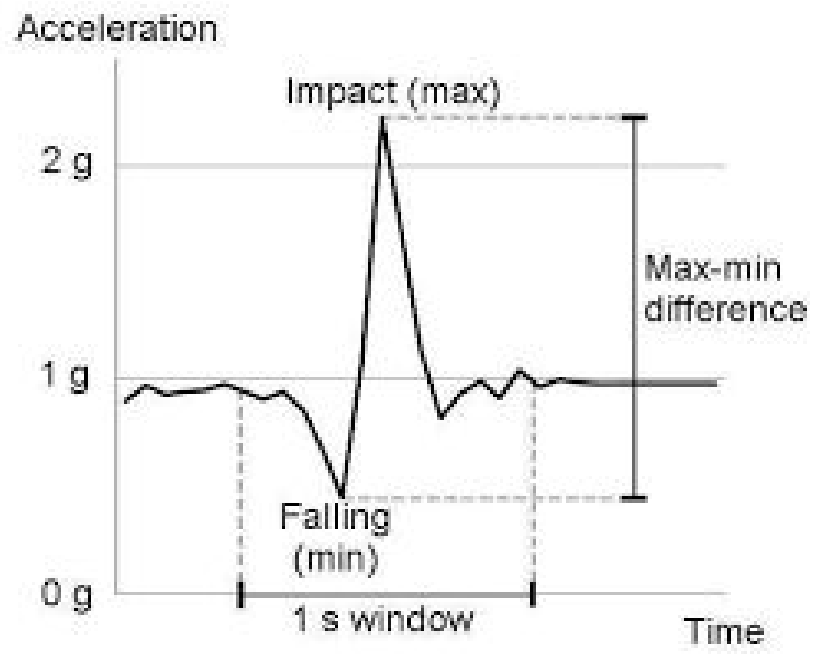

Figure 4. Graph of fall.

\section{Principle component analysis}

The principle component analysis is a most successful and frequent technique used in the feature extraction process. It is mathematical principle that transforms a huge dataset into a smaller number of variables. The linear discriminant analysis (LDA) and principle component analysis are linear transformations methods. PCA projects the entire dataset onto a different feature (sub) space, and LDA tries to determine a suitable feature (sub) space in order to distinguish between patterns that belong to different classes.

\section{Summary of the PCA approach}

- Standardize the data

- Obtain the Eigenvectors and Eigen values from the covariance matrix or correlation matrix, or perform singular vector decomposition.

- Sort Eigen values in descending order and choose the $k k$ eigenvectors that correspond to the $k k$ largest eigenvalues where $k k$ is the number of dimensions of the new feature subspace $(k \leq d k \leq d)$.

- Construct the projection matrix $W W$ from the selected $k k$ eigenvectors.

- Transform the original dataset $X X$ via $W W$ to obtain a $k k$ dimensional feature subspace $Y Y$.

\section{Singular value decomposition}

Singular value decomposition takes a rectangular matrix of gene expression data (defined as $\mathrm{A}$, where $\mathrm{A}$ is a $\mathrm{n} \times \mathrm{p}$ matrix) in which the $n$ rows represents the genes, and the $p$ columns represents the experimental conditions. The SVD theorem states:

$A_{\mathrm{n} \times \mathrm{p}}=U_{\mathrm{n} \times \mathrm{n}} S_{\mathrm{n} \times \mathrm{p}} V_{\mathrm{p} \times \mathrm{p}}^{\mathrm{T}} \rightarrow(4)$

Where

$\mathrm{UTU}=I_{\mathrm{n} \times \mathrm{n}}$

$\mathrm{VTV}=I_{\mathrm{p} \times \mathrm{p}}($ i.e. $\mathrm{U}$ and $\mathrm{V}$ are orthogonal)

Where the columns of $U$ are the left singular vectors; $S$ has singular values and is diagonal; and VT has rows that are the right singular vectors. The SVD represents an expansion of the original data in a coordinate system where the covariance matrix is diagonal. Calculating the SVD consists of finding the eigenvalues and eigenvectors of AAT and ATA. The eigenvectors of ATA make up the columns of $\mathrm{V}$, the eigenvectors of AAT make up the columns of U. Also, the singular values in $\mathrm{S}$ are square roots of eigenvalues from AAT or ATA. The singular values are the diagonal entries of the S matrix and are arranged in descending order. The singular values are always real numbers. If the matrix $\mathrm{A}$ is a real matrix, then $U$ and $V$ are also real.

\section{Hybrid classification}

The classification algorithm is identified which is best suited for the output of feature extraction.

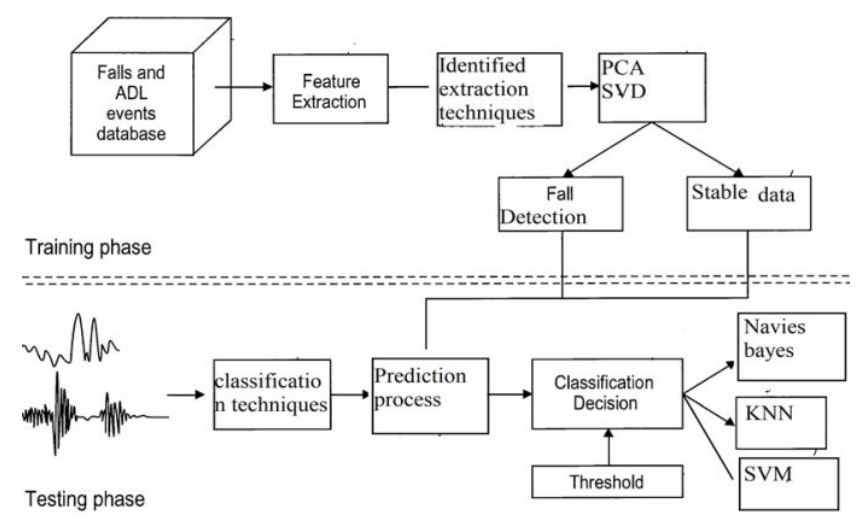

Figure 5. Proposed architecture.

The proposed technique in the classification process focus on the hybrid algorithm. We analyse the data set with the feature extraction technique and design a feature extraction technique for the data sets to develop a classification algorithm. It also evaluates the efficiency of these algorithms, and comparison is made on the accuracy. They are several algorithms for the process of classification of data in machine learning. The feature extraction focused on the principle component analysis and singular value decomposition as shown above.

The Figure 5 shows the architecture of the proposed system it depicts the training and the testing phase of the different datasets. The hybrid algorithm focuses on the accuracy of the data identified and the efficiency is calculated by comparing with the existing classification technique. 


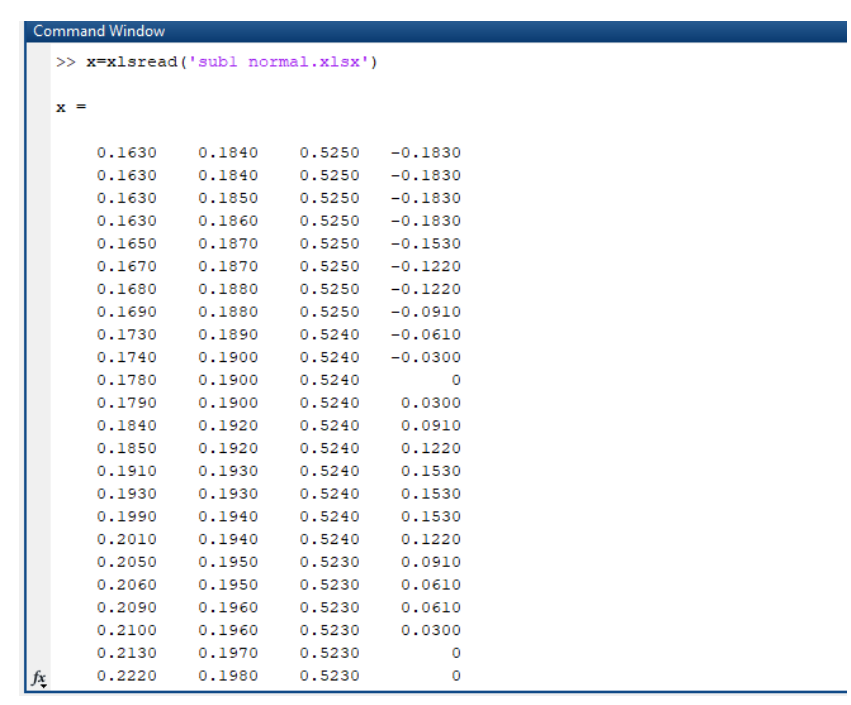

Figure 6. Input data.

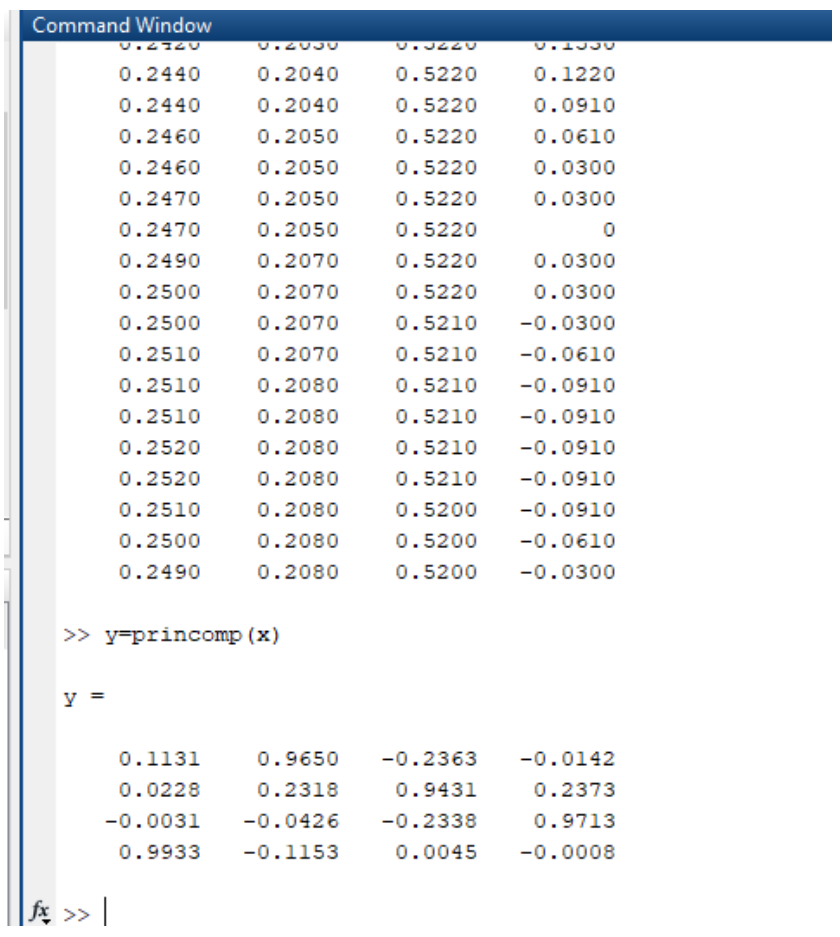

Figure 7. Reduced data set.

\section{Experimental Discussion}

In this work, the data set is collected from the publicly available benchmark data. The dataset consists of both the normal and the abnormal data of elderly person. Normal data means data taken when there is usual movement by the elderly sitting walking etc. Abnormal means data taken when there is an unusual bending, falling or rolling etc. This dataset is considered as distributed dataset since it contains both normal and abnormal values. Data set has both normal and abnormal values. The abnormal data set is identified by the values as they differ much from the normal dataset. Figure 6 shows the input data read from.csv in Matlab. Figure 7 shows the data after feature extraction.
The input data is read in MATLAB using the syntax to SA the process of feature extraction. Once the data are read the principle component analysis and the singular value decomposition feature extraction techniques are used to the processing of removing the unwanted and redundant data. The reduced data set are used to determine the difference values between the normal and abnormal data. To find the exact difference the values are plotted in graph that is shown in Figure 8. It describes only one sample of the plotting of graph of the normal and abnormal data of different subjects (Figure 9). The below figure (Figure 10) shows the ROC curve that represents the true positive and the negative rates of the classification algorithm. The rates are compared with the existing technique and the efficiency is calculated.

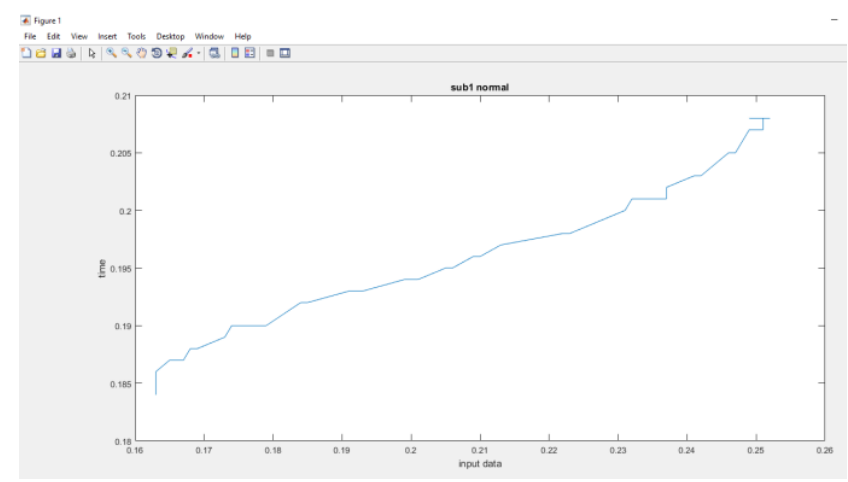

Figure 8. Plotting of data.

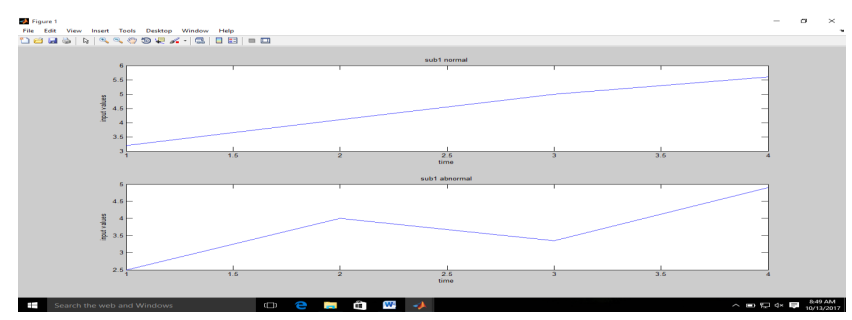

Figure 9. Normal and abnormal graph.

The threshold value is fixed based on the plotted datasets of the normal and the abnormal data (Figure 11). The value shows the accuracy of the fall data.

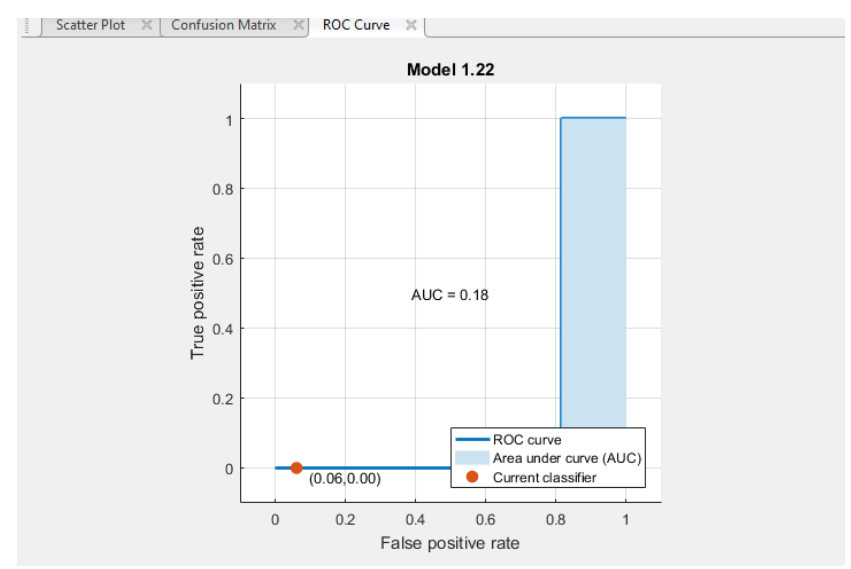

Figure 10. ROC curve. 


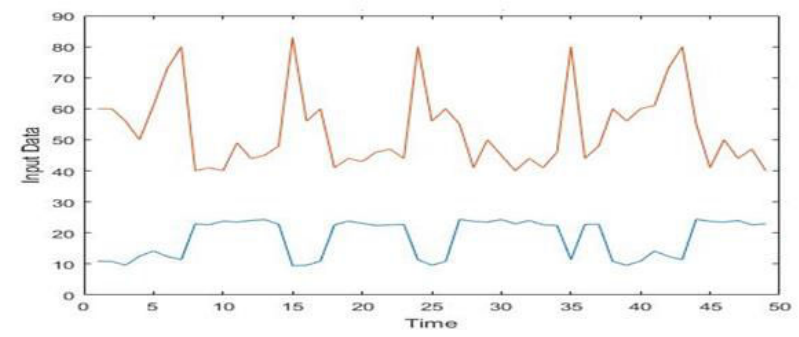

Figure 11. Plotted dataset.

\section{Conclusion}

The experiment proved that the proposed system takes advantage of wearable devices which are able to detect simulated falls with sufficient accuracy, and can provide timely and accurate help for the elderly. Based on the results achieved, some sensor boards, along with the software for fall detection, can be contributed to some elderly communities, so as to collect data for the daily living activities of the elderly, and harvest the database of real-world falls. Based on the accuracy of recognition rate and computational complexity, the series of methods of surface EMG feature extraction and recognition were estimated for activity monitoring and fall detection.

The subject was undertaken according to two research tracks: (1) fall detection and (2) fall prevention. This gives a basic understanding on elderly falls, their intrinsic and extrinsic causes and their consequences. Fall detection systems were summarized and organized with respect to their sensor deployment and their data processing techniques. Fall prevention systems were also surveyed; however more details were accorded to existing solutions given the current efforts in this research track. Further in exporting gait and balance assessment tools into homes while providing reliable and low cost solutions for the elders. In this paper the performance and computational requirements of the machine learning techniques are compared based on the same dataset and achieve accuracies. In fall incident detection, the system makes use of KNN to classify human body postures and the best $\mathrm{k}$ value in $\mathrm{KNN}$ algorithm is determined by $\mathrm{k}$-fold cross-validation scheme.

\section{References}

1. Sapsford R, Jupp V. Data collection and analysis (2nd Edn.). SAGE Publications Ltd.: London, UK, 2006.

2. Luštrek M, Kaluža B. Fall detection and activity recognition with machine learning. Informatica 2009; 33: 205-212.

3. Pecchia L, Melillo P, Bracale M. Remote health monitoring of heart failure with data mining via CART method on HRV features. IEEE Trans Biomed Eng 2011; 58: 800-804.

4. Perry J, Kellog S, Vaidya S, Youn JH, Ali H, Sharif H. Survey and evaluation of real-time fall detection approaches. High-Capacity Optical Networks and
Enabling Technologies (HONET), 2009 6th International Symposium 2009; 158-164.

5. Duda RO, Hart PE, Stork DG. Pattern classification (2nd Edn.). John Wiley \& Sons Inc. New York NY USA 2001.

6. Ghadimi N, Mohammad O. A novel design of low power rectenna for wireless sensor and RFID applications. Wireless Personal Commun 2014; 78.

7. Huang CN, Chiang CY, Chen GC, Hsu S, Chu WC, Chan CT. Fall detection system for healthcare quality improvement in residential care facilities. J Med Biol Eng 2010; 30: 247-252.

8. Bourke AK, Lyons GM. A threshold-based fall-detection algorithm using a bi-axial gyroscope sensor. Med Eng Phys 2008; 30: 84-90.

9. Chao PK, Chan HL, Tang FT, Chen YC, Wong MK. A comparison of automatic fall detection by the crossproduct and magnitude of tri-axial acceleration. Physiol Meas 2009; 30: 1027-1037.

10. Razmjooy N, Mehdi R, Noradin G. Imperialist competitive algorithm-based optimization of neuro-fuzzy system parameters for automatic red-eye removal. IntJ Fuzzy Sys 2017; 19: 1-13.

11. Mubashir M, Shao L, Seed L. A survey on fall detection: principles and approaches. Neurocomputing 2013; 100: 144-152.

12. Orces $\mathrm{CH}$, Alamgir H. Trends in fall-related injuries among older adults treated in emergency departments in the USA. Inj Prev 2014; 20: 421-423.

13. Ramachandran R, Ramanna L, Ghasemzadeh H, Pradhan G, Jafari R, Prabhakaran B. Body sensor networks to evaluate standing balance: interpreting muscular activities based on inertial sensors. Proceedings of the 2nd International Workshop on Systems and Networking Support for Health Care and Assisted Living Environments, Breckenridge, CO, USA 2008.

14. Yang X, Dinh A, Chen L. Implementation of a wearable real-time system for physical activity recognition based on Naive Bayes classifier. International Conference on Bioinformatics and Biomedical Technology, China 2010.

15. Sulistijono Z, Ardiansyah IA. Comparison of five time series EMG features extractions using Myo Armband. Proceedings of the 2015 International Electronics Symposium (IES), Surabaya, Indonesia 2015; 11-14.

16. Zhang T, Wang J, Xu L, Liu P. Fall detection by wearable sensor and one-class SVM.Sem Schol 2006.

17. Zhang Z, Conly C, Athitsos V. A survey on vision-based fall detection. Proceedings of the 8th International Conference on Pervasive Technologies Related to Assistive Environments ACM 2015.

18. Zhang Q, Tian G, Ding N, Zhang Y. A fall detection study based on neural network algorithm using AHRS. IEEE Inform Autom 2013; 773-779.

19. Melillo P, Jovic A, De Luca N, Pecchia L. Automatic classifier based on heart rate variability to identify fallers among hypertensive subjects. Healthcare Technol Lett 2015; 2: 89-94. 
20. Razmjooy N, Sheykhahmad FR, Ghadimi N. A hybrid neural network-world cup optimization algorithm for melanoma detection. Open Med (Wars) 2018; 13: 9-16.

21. Pannurat N, Thiemjarus S, Nantajeewarawat E. Automatic fall monitoring: a review. Sensors (Basel) 2014; 14: 12900-12936.

22. Rimminen H, Lindström J, Linnavuo M, Sepponen R. Detection of falls among the elderly by a floor sensor using the electric near field. IEEE Trans Inf Technol Biomed 2010; 14: 1475-1476.

23. Zhang Z, Su J. Naive Bayes for optimal ranking. J Exp Theor Artif Intell 2008; 20: 79-93.

24. Parsian A, Mehdi R, Noradin G. A hybrid neural networkgray wolf optimization algorithm for melanoma detection. Biomed Res 2017; 28.

25. Tinetti ME, Williams CS. Falls, injuries due to falls, and the risk of admission to a nursing home. N Engl J Med 1997; 337: 1279-1284.
26. Yoshida S. A global report on falls prevention epidemiology of falls. World Health Organization Geneva, Switzerland 2007.

27. Yang CC, Hsu YL. A review of accelerometry-based wearable motion detectors for physical activity monitoring. Sensors 2010; 10: 7772-7788.

\section{*Correspondence to}

Sheryl Oliver A

St. Joseph's College of Engineering

Chennai

India 